\title{
Layered spatial modulation
}

\author{
Salim Abdelkareem Alkhawaldeh
}

\begin{abstract}
In this paper, we propose a layered spatial modulation (L-SM) scheme that combines a multi-layered coding scheme with the spatial modulation. At each antenna selection, the number of active transmit antennas equals the number of layers. The proposed L-SM scheme maximizes the diversity order and coding gain at each antenna selection. The exact pairwise and upper bound bit error probabilities are derived. Several examples of different spatial modulation schemes and the proposed L-SM scheme are simulated with the same number of transmit antennas and same spectral efficiency. As expected, the proposed L-SM scheme satisfies significant increase in the performance compared to all existing spatial modulation schemes.
\end{abstract}

Keywords: MIMO, Diversity, Spatial multiplexing, Layered spatial modulation, STBC, SM, L-SM

\section{Introduction}

Significant improvements in the bandwidth efficiency and notable reduction in the probability of error can be obtained by using multiple input multiple output (MIMO) technology $[1,2]$. This technology uses multiple transmit and multiple receive antennas where space and time diversities and spatial multiplexing are applied. Several space-time trellis and block coding schemes that improve the diversity and coding gains were proposed [3-9]. On the other hand, a number of coding schemes were introduced to improve the spatial multiplexing gain [10-17]. All the above schemes use more than one transmit antenna in the same time. Simultaneous activation of multiple transmit antennas causes the inter-channel interference. To avoid this problem, spatial modulation (SM) scheme was presented $[18,19]$. This scheme uses one transmit antenna at each time period depending on the first group of input bits. The second group of bits is used to determine which symbol is selected from the constellation set. Unfortunately, this scheme needs large number of transmit antennas to increase the spectral efficiency of wireless system. To reduce the number of transmit antennas, generalized spatial modulation scheme was proposed [20]. This scheme uses more than one transmit antenna at each time period. However, the transmit diversity is not exploited in this scheme which yields significant degradation in the performance. To overcome this problem, a

\footnotetext{
Correspondence: skhawaldeh@bau.edu.jo; skhawaldeh@yahoo.com Communication Engineering Department, Faculty of Engineering Technology, Albalqa Applied University, Amman, Jordan
}

scheme that combines spatial modulation and space-time block coding (STBC) referred as STBC-SM was proposed [21]. This scheme takes the advantages of both spatial modulation and space-time block coding. Also, it maximizes the diversity order and multiplexing gain. This scheme suffers from degradation of the spectral efficiency offered by the spatial dimension compared with the conventional spatial modulation. To improve the spectral efficiency, high-rate STBC-SM (H-STBC-SM) scheme and spatial modulation STBC scheme with cyclic structure (STBC-CSM) were introduced [22, 23]. The number of codewords provided by these schemes is twice the number of codewords provided by the STBC-SM in [21]. The transmit diversity order achieved by both schemes is equal to two. However, the H-STBC-SM suffers from small degradation in the performance. At the same spectral efficiency and with transmit antennas less than those of the STBC-SM scheme, the performance of STBC-CSM approaches that of the STBC-SM. The authors in [24] proposed the complex interleaved orthogonal design SM with the high degree of spatial modulation (CIOD-SM-H). The spectral efficiency of this scheme is as that of the $\mathrm{H}$-STBC-SM scheme, and its performance is less than that of the STBC-SM. To further increase the spectral efficiency and the performance, cyclic temporally and spatially modulated STBC (STBC-TSM) scheme was proposed [25]. In this scheme, Alamouti STBC is rotated over four symbol periods to ensure a transmit diversity order of 2 .

In this paper, we propose a layered spatial modulation (L-SM) scheme that avoids large number of transmit 
antennas and provides high spectral efficiency. The proposed L-SM scheme achieves significant improvement in the performance compared to that of the all above spatial modulation schemes. The proposed L-SM scheme is based on a multi-layered coding scheme combined with the spatial modulation scheme in $[18,19]$. At each antenna selection, the proposed L-SM scheme is used with transmit diversity order equals the number of active transmit antennas and maximum coding gain based on specific codeword matrices. The number of layers of the proposed scheme is equal to the number of active transmit antennas at each antenna selection.

The exact pairwise and upper bound bit error probabilities of the proposed L-SM scheme are derived. Simulation results are demonstrated with several examples of the proposed L-SM scheme and recent spatial modulation schemes combined with STBC. All schemes are compared with the same number of transmit antennas and comparable spectral efficiency. These results show that the proposed L-SM scheme significantly outperforms all other spatial modulation schemes.

This paper is organized as follows: In Section 2, the methods that we used are presented. Conventional spatial modulation scheme is presented in Section 3. In Section 4, we propose the L-SM scheme. We derive the pairwise and bit error probabilities of the proposed L-SM scheme in Section 5. In Section 6, numerical results of the proposed L-SM and different spatial modulation schemes are demonstrated. Finally, the conclusions of this paper are inserted in Section 7.

\section{Methods}

The proposed L-SM scheme is a combination of a multi-layered coding scheme and the spatial modulation. The codeword of the multi-layered coding scheme contains a number of layers with the same number of active transmit antennas. A number of phase shifts are inserted in the codeword among the layers and symbols to maximize the diversity and coding gains. When all transmit antennas are active, one codeword is transmitted over one codeword period. When the total number of transmit antennas is greater than or equals twice the number of active transmit antennas, two codewords are transmitted from different active transmit antennas over two different codeword periods at each antenna selection case. Several examples of the proposed L-SM and the state-of-the-art schemes are simulated using Matlab software. The channel is modeled by using the Monte Carlo method. The schemes are compared to each other with the same number of transmit and receive antennas and same spectral efficiency.

\section{Conventional spatial modulation scheme}

The SM scheme in $[18,19]$ is described in this section as shown in Fig. 1. This scheme uses two groups of bits. The first group of $k$ bits is used to select one active transmit antenna $i$ out of $N_{\text {all }}$ antennas at each time period, and the second group of $u$ bits is used to select the symbol $c$ from the constellation set that has $2^{u}$ elements. This results in transmission rate of $(k+u)$ bits per channel use. The advantage of this approach is that it avoids the problem of inter-channel interference.

The signal at receive antenna $j$ can be written as

$$
y_{j}=\sqrt{E_{s}} a_{j i} c_{i}+v_{j}
$$

where $E_{s}$ is the average symbol energy, $c_{i}$ is the symbol $c$ transmitted from antenna $i$, and $a_{j i}$ is the path gain between transmit antenna $i$ and receive antenna $j$. It is assumed that the path gains are samples of a zero mean complex Gaussian random variable with variance of 0.5 per dimension. The additive noise $v_{j}$ at the receive antenna $j$ is assumed to be independent samples of a zero mean complex Gaussian random variable with variance of $N_{0} / 2$ per dimension.

The exact pairwise error probability of transmitting $c_{i}$ and deciding $\tilde{c}_{\tilde{i}}$ is given by [20]

$$
\begin{aligned}
P_{r}\left(c_{i} \rightarrow \tilde{c}_{i}\right) & =I_{\frac{N_{0}}{N_{0}+\tilde{N}_{0}}}\left(N_{r}, N_{r}\right) \quad i \neq \tilde{i}, \quad i \\
& =1,2, \cdots, N_{t}, \tilde{i}=1,2, \cdots, N_{t}
\end{aligned}
$$

where $\tilde{N}_{0}=N_{0}+E_{s}|c|^{2}+E_{s}|\tilde{c}|^{2}$ and $I_{x}\left(w_{1}, w_{2}\right)$ is the regularized beta function given by

$$
I_{x}\left(w_{1}, w_{2}\right)=\frac{1}{R\left(w_{1}, w_{2}\right)} \int_{t=0}^{x} t^{\left(w_{1}-1\right)}(1-t)^{\left(w_{2}-1\right)} d t
$$

with

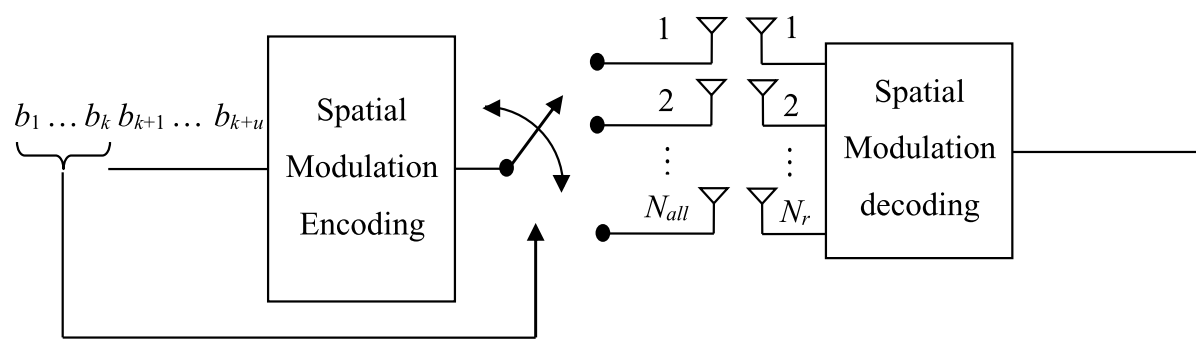

Fig. 1 Block diagram of the conventional spatial modulation scheme [18, 19] 


$$
R\left(w_{1}, w_{2}\right)=\int_{t=0}^{1} t^{\left(w_{1}-1\right)}(1-t)^{\left(w_{2}-1\right)} d t
$$

The bit error probability of spatial modulation scheme is given by

$$
P_{\mathrm{bit}} \leq \sum_{c, i} \sum_{\tilde{c}, \tilde{i}} \frac{N\left(c_{i}, \tilde{c}_{\tilde{i}}\right) I_{\frac{N_{0}}{N_{0}+\tilde{N}_{0}}}\left(N_{r}, N_{r}\right)}{(k+u) 2^{k+u}}
$$

where $N\left(c_{i}, \tilde{c}_{\tilde{i}}\right)$ is the number of bits in error between $c_{i}$ and $\tilde{c}_{\tilde{i}}$

As an example of this scheme is to apply 3 bits per channel use. The first 2 bits are used to select the active transmit antenna out of four antennas, and the third bit is used to select the symbol from the binary phase shift keying (BPSK) constellation set. The transmission and encoding of this example is shown in Table 1.

\section{Proposed L-SM scheme}

\subsection{Proposed multi-layered coding scheme without spatial modulation}

In this subsection, we present a multi-layered coding scheme that maximizes the diversity order and spatial multiplexing gain as shown in Fig. 2.

In this scheme, multi layers with the same number of transmit antennas are applied. Each layer contains a number of symbols. The received signal matrix is given as

$$
\mathbf{Y}=\sqrt{\frac{E_{s}}{N_{t}}} \mathbf{A C}+\mathbf{V}
$$

where $\mathbf{V}$ is $N_{r} \times N_{t}$ noise matrix at the receive antennas whose entries are independent samples of a zero mean complex Gaussian random variable with variance of $N_{0} / 2$ per dimension. A is $N_{r} \times N_{t}$ channel matrix whose entry $a_{j i}$ is the path gain between transmit antenna $i$ and receive antenna $j$, and it is given as

Table 1 The transmission and encoding of SM scheme with 3 bits/channel use, four transmit antennas, and BPSK modulation

\begin{tabular}{lll}
\hline $\begin{array}{l}\text { Message bits } \\
b_{1} b_{2} b_{3}\end{array}$ & Active antennas & $\begin{array}{l}\text { Transmitted symbol } \\
c\end{array}$ \\
\hline 000 & 1 & -1 \\
010 & 2 & -1 \\
100 & 3 & -1 \\
110 & 4 & -1 \\
001 & 1 & 1 \\
011 & 2 & 1 \\
101 & 3 & 1 \\
111 & 4 & 1 \\
\hline
\end{tabular}

$$
\mathbf{A}=\left[\begin{array}{llll}
a_{11} & a_{12} & \cdots & a_{1 N_{t}} \\
a_{21} & a_{22} & \cdots & a_{2 N_{t}} \\
\vdots & & & \\
a_{N_{r} 1} & a_{N_{r}} & \cdots & a_{N_{r} N_{t}}
\end{array}\right]
$$

It is assumed that the path gains in $\mathbf{A}$ are samples of a zero mean complex Gaussian random variable with variance of 0.5 per dimension. In (6), $\mathbf{C}$ is the codeword matrix given by

$$
\begin{aligned}
& \mathbf{C}=\left[\begin{array}{llll}
s_{1,1} & s_{2,1} & \cdots & s_{N_{t}, 1} \\
s_{N_{t}, 2} & s_{1,2} & \cdots & s_{N_{t}-1,2} \\
\vdots & & & \\
s_{2, N_{t}} & \cdots & s_{1, N_{t}}
\end{array}\right] \\
& s_{i, t}=\left[\begin{array}{llll}
c_{1, i} & c_{2, i} & \cdots & c_{N_{t}, i}
\end{array}\right] \mathbf{p}^{j \alpha_{i}}, i=1,2, \cdots, N_{t}, \quad t=1,2, \cdots, N_{t} \\
& \mathbf{p}=\left[\begin{array}{llll}
e^{j \beta_{1}} & e^{-j \frac{2 \pi(t-1)}{N_{t}}} & e^{j \beta_{2}} & e^{-j \frac{4 \pi(t-1)}{N_{t}}} e^{j \beta_{3} \cdots e^{-j \frac{j\left(N_{t}-1\right) \pi(t-1)}{N_{t}}}} e^{j \beta_{N_{t}}}
\end{array}\right]
\end{aligned}
$$

where $c_{j, i}$ is the $i$ th symbol of the $j$ th layer. The symbols $c_{j, i} s$ are selected by the bits $b_{1} b_{2} \ldots b_{u}$. In (9) and (10), $\alpha_{1}, \alpha_{2}, \cdots, \alpha_{N_{t}}$ and $\beta_{1}, \beta_{2}, \cdots, \beta_{N_{t}}$ are the phase shifts among transmitted symbols and layers, respectively. It is worthy of noting that this scheme provides spectral efficiency of $N_{t}$ symbols per channel use.

The upper bound of the average probability of codeword matrix $\mathbf{C}_{m}$ and the decoder decided $\mathbf{C}_{n}$ is given in [13] as

$$
P\left(\mathbf{C}_{m} \rightarrow \mathbf{C}_{n}\right) \leq \frac{1}{\left|\mathbf{I}_{N_{r} \times N_{r}}+\frac{E_{s}}{4 N_{0} N_{t}} \mathbf{R} \otimes \mathbf{I}_{N_{r}}\right|}
$$

where $\mathbf{R}=\left(\mathbf{C}_{m}-\mathbf{C}_{n}\right)\left(\mathbf{C}_{m}-\mathbf{C}_{n}\right)^{H}$ and the superscript $H$ denotes Hermitian transpose. This result was derived by using the Chernoff's upper bound [26].

In the case of high signal to noise ratio, (11) can be written as

$$
P\left(\mathbf{C}_{m} \rightarrow \mathbf{C}_{n}\right) \leq\left(\lambda_{1} \lambda_{2} \cdots \lambda_{r}\right)^{-N_{r}}\left(\frac{E_{s}}{4 N_{0} N_{t}}\right)^{-r N_{r}}
$$

where $r$ and $\lambda_{1}, \lambda_{2}, \cdots, \lambda_{r}$ are the rank and the eigenvalues of matrix $\mathbf{R}$, respectively.

The angles $\alpha_{1}, \cdots, \alpha_{N_{t}}, \beta_{1}, \cdots, \beta_{N_{t}}$ have to be chosen to increase the upper bound of (11). This can be obtained by maximizing the rank $r$ to become $N_{t}$ and the product of eigenvalues $\left(\lambda_{1} \lambda_{2} \cdots \lambda_{r}\right)$ of $\mathbf{R}$.

It is worthy of noting that this coding scheme achieves diversity order of $N_{t} \times N_{r}$ and maximum coding gain $\left(\lambda_{1} \lambda_{2} \cdots \lambda_{N_{t}}\right)^{\frac{1}{N_{t}}}$ under specific values of $\alpha_{1}, \cdots, \alpha_{N_{t}}, \beta_{1}, \cdots, \beta_{N_{t}}$. 


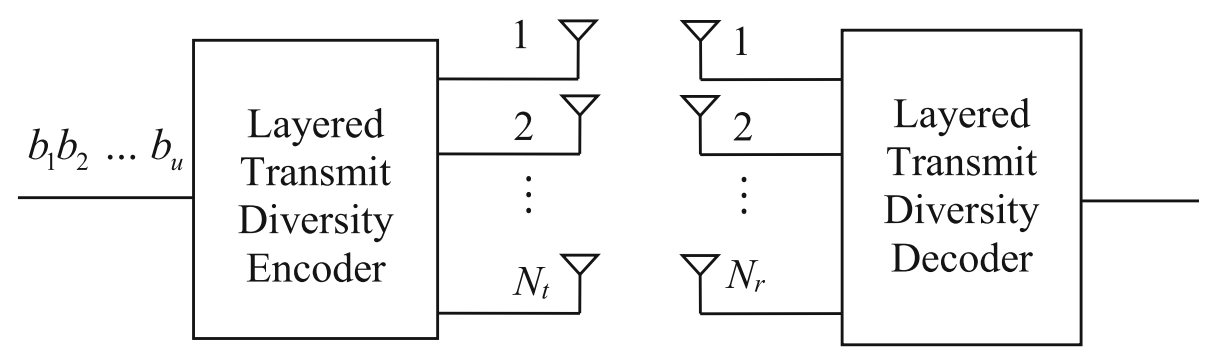

Fig. 2 Layered coding scheme

\subsection{Proposed L-SM scheme}

In this section, we combine the proposed layered coding scheme presented in Section 4.1 with the spatial modulation scheme presented in $[18,19]$ to form a layered spatial modulation (L-SM) scheme. Assume that the total number of antennas $=N_{\text {all }}$ and the number of active transmit antennas at each antenna selection $=N_{t}$. In Section 4.1, $N_{\text {all }}=N_{t}$ and one codeword matrix is transmitted over $N_{t}$ symbol periods (one codeword period) with diversity order equals $N_{t} \times N_{r}$. In this case, there is no antenna selection and the proposed scheme uses $N_{t}^{2}$ symbols per $N_{t}$ channel use. For $N_{\text {all }} \geq 2 N_{t}$, two different codewords are transmitted from different active transmit antennas over two different codeword periods at each antenna selection case. Depending on the antenna selection case, each codeword is transmitted circularly from $N_{t}$ active antennas along the total number of transmit antennas. This produces $N=\frac{2 N_{\text {all }} !}{\left(N_{\text {all }}-N_{t}\right) ! N_{t} !}+N_{\text {all }}$ antenna selections and the number of bits related to these selections $=k=\log _{2} N$. The proposed L-SM scheme achieves diversity order of $N_{t} \times N_{r}$ and maximum coding gain at each case of the antenna selections. Since each codeword contains $N_{t}^{2}$ symbols, the proposed L-SM scheme uses ( $k$ bits $+2 N_{t}^{2}$ symbols) per $2 N_{t}$ channel use which is equivalent to $\left(\frac{k}{2 N_{t}}\right.$ bits $+N_{t}$ symbols) per channel use. Note that $N_{t}$ symbols $=N_{t} \log _{2} M$ bits. This yields $\left(\frac{k}{2 N_{t}}+N_{t} \log _{2} M\right)$ bits per channel use. For the proposed L-SM scheme, the received signal matrix over the two codeword periods is sent to the maximum likelihood decoder to estimate the $k$ bits $\left(b_{1}, b_{2}, \ldots, b_{k}\right)$ related to antenna selections and the $2 N_{t}^{2} \log _{2} M$ bits related to the symbols in the two codeword matrices. Two different examples are introduced to explain the concept of the L-SM scheme.

Example 1 We consider the proposed scheme with $N_{\text {all }}=N_{t}=2$. In this case, there is no antenna selection. Therefore, one codeword with two layers is transmitted from two antennas over one codeword period of time. Assume that the symbols $c_{11}$ and $c_{12}$ are related to layer 1 and the symbols $c_{21}$ and $c_{22}$ are related to layer 2 . According to (8), (9), and (10), the transmitted codeword matrix $\mathbf{C}$ of this example is given by

$$
\mathbf{C}=\left[\begin{array}{ll}
e^{j \alpha_{1}}\left(e^{j \beta_{1}} c_{11}+e^{j \beta_{2}} c_{21}\right) & e^{j \alpha_{2}}\left(e^{j \beta_{1}} c_{12}+e^{j \beta_{2}} c_{22}\right) \\
e^{j \alpha_{2}}\left(e^{j \beta_{1}} c_{12}-e^{j \beta_{2}} c_{22}\right) & e^{j \alpha_{1}}\left(e^{j \beta_{1}} c_{11}-e^{j \beta_{2}} c_{21}\right)
\end{array}\right]
$$

The spectral efficiency of this case is equal to 2 symbols $/ \mathrm{s} / \mathrm{Hz}=\left(2 \log _{2} M\right)$ bits $/ \mathrm{s} / \mathrm{Hz}$. For the case of BPSK modulation, we assume that $\alpha_{1}=\beta_{1}=0$ as reference. The phase shifts $\alpha_{2}$ and $\beta_{2}$ have to be chosen to increase the upper bound of (11). This can be obtained by maximizing the rank $r$ and the product of eigenvalues $\left(\lambda_{1} \lambda_{2}\right)$ of $\mathbf{R}$. A rank $r$ of 2 and maximum product of eigenvalues $\left(\lambda_{1} \lambda_{2}\right)$ occur when $\alpha_{2}=\beta_{2}=\frac{\pi}{2}$. For the case of quadrature phase shift keying (QPSK) modulation, $\alpha_{1}=\beta_{1}=0, \alpha_{2}=\frac{\pi}{6}$, and $\beta_{2}=\frac{\pi}{4}$ and the spectral efficiency $=4 \mathrm{bits} / \mathrm{s} / \mathrm{Hz}$. For the case of 8-QAM modulation, $\alpha_{1}=\beta_{1}=0, \alpha_{2}=0.21 \pi$, and $\beta_{2}=\frac{3 \pi}{16}$ and the spectral efficiency $=6 \mathrm{bits} / \mathrm{s} / \mathrm{Hz}$. For the case of 16-QAM modulation, $\alpha_{1}=\beta_{1}=0, \alpha_{2}=0.1 \pi$, and $\beta_{2}=\frac{\pi}{4}$ and the spectral efficiency $=8 \mathrm{bits} / \mathrm{s} / \mathrm{Hz}$.

Example 2 We consider the proposed L-SM scheme with $N_{\text {all }}=4, N_{t}=2$ and 2 transmitted codewords $C$ and E. The transmission approach of this example is demonstrated in Table 2. Note that the two codewords are transmitted from different active transmit antennas over two different codeword periods at each antenna selection case. The rotation of the two codeword matrices is different than that in [21-25]. The number of antenna selections $N=\frac{2 \times 4 !}{(4-2) ! 2 !}+4=16$ as shown in Table 2 and $k=4$. For BPSK, QPSK, 8-QAM, and 16-QAM modulation, the angles $\alpha_{1}, \beta_{1}, \alpha_{2}$, and $\beta_{2}$ are the same as those in Example 1 whereas the angles $\theta_{1}, \theta_{2}, \theta_{3}, \theta_{4}$, and $\theta_{5}$ are selected to maximize the diversity order to $2 N_{r}$ and multiplexing gain and they are equal to $\frac{\pi}{12}, \frac{2 \pi}{12}, \frac{4 \pi}{12}, \frac{5 \pi}{12}$, and $\frac{3 \pi}{12}$, respectively. The spectral efficiency of this example $=\left(\frac{k}{2 N_{t}}\right.$ bits $+N_{t}$ symbols $)$ per channel use $=\left(1+2 \log _{2} M\right)$ bits/s/Hz.

\section{Performance analysis of the proposed L-SM scheme}

The performance of the proposed L-SM scheme is derived in this section. At antenna selection related to $b_{1}$, $b_{2}, \ldots, b_{k}$, the received signal matrices $\mathbf{Y}_{m}$ and $\mathbf{Y}_{p}$ related 
Table 2 The transmission and encoding of the proposed L-SM scheme with $N_{\text {all }}=4, N_{t}=2$, and $\left(1+2 \log _{2} M\right)$ bits $/ \mathrm{s} / \mathrm{Hz}$

\begin{tabular}{|c|c|c|c|c|c|c|c|}
\hline \multicolumn{4}{|c|}{ The first codeword period } & \multicolumn{4}{|c|}{ The second codeword period } \\
\hline Ant.1 & Ant.2 & Ant.3 & Ant.4 & Ant.1 & Ant.2 & Ant.3 & Ant.4 \\
\hline$C(1,1)$ & $C(2,1)$ & 0 & 0 & 0 & 0 & $E(1,1)$ & $E(2,1)$ \\
\hline$C(1,2)$ & $C(2,2)$ & 0 & 0 & 0 & 0 & $E(1,2)$ & $E(2,2)$ \\
\hline 0 & 0 & $C(1,1)$ & $C(2,1)$ & $E(1,1)$ & $E(2,1)$ & 0 & 0 \\
\hline 0 & 0 & $C(1,2)$ & $C(2,2)$ & $E(1,2)$ & $E(2,2)$ & 0 & 0 \\
\hline$C(1,1) e^{j \theta_{1}}$ & 0 & $C(2,1) e^{j \theta_{1}}$ & 0 & 0 & $\mathrm{E}(1,1) e^{j \theta_{2}}$ & 0 & $E(2,1) e^{j \theta_{2}}$ \\
\hline$C(1,2) e^{j \theta_{1}}$ & 0 & $C(2,2) e^{j \theta_{1}}$ & 0 & 0 & $E(1,2) e^{j \theta_{2}}$ & 0 & $E(2,2) e^{j \theta_{2}}$ \\
\hline 0 & $C(1,1) e^{j \theta_{2}}$ & 0 & $C(2,1) e^{j \theta_{2}}$ & $\mathrm{E}(1,1) e^{j \theta_{1}}$ & 0 & $E(2,1) e^{j \theta_{1}}$ & 0 \\
\hline 0 & $C(1,2) e^{j \theta_{2}}$ & 0 & $C(2,2) e^{j \theta_{2}}$ & $\mathrm{E}(1,2) e^{j \theta_{1}}$ & 0 & $E(2,2) e^{j \theta_{1}}$ & 0 \\
\hline$C(1,1) e^{j \theta_{3}}$ & 0 & 0 & $C(2,1) e^{j \theta_{3}}$ & 0 & $E(1,1) e^{j \theta_{4}}$ & $E(2,1) e^{j \theta_{4}}$ & 0 \\
\hline$C(1,2) e^{j \theta_{3}}$ & 0 & 0 & $C(2,2) e^{j \theta_{3}}$ & 0 & $E(1,2) e^{j \theta_{4}}$ & $E(2,2) e^{j \theta_{4}}$ & 0 \\
\hline 0 & $C(1,1) e^{j \theta_{4}}$ & $C(2,1) e^{j \theta_{4}}$ & 0 & $\mathrm{E}(1,1) e^{j \theta_{3}}$ & 0 & 0 & $E(2,1) e^{j \theta_{3}}$ \\
\hline 0 & $C(1,2) e^{j \theta_{4}}$ & $C(2,2) e^{j \theta_{4}}$ & 0 & $E(1,2) e^{j \theta_{3}}$ & 0 & 0 & $E(2,2) e^{j \theta_{3}}$ \\
\hline$C(1,1) e^{j \theta_{5}}$ & $C(2,1) e^{j \theta_{5}}$ & 0 & 0 & 0 & 0 & $E(1,1) e^{j \theta_{5}}$ & $E(2,1) e^{j \theta_{5}}$ \\
\hline$C(1,2) e^{j \theta_{5}}$ & $C(2,2) e^{j \theta_{5}}$ & 0 & 0 & 0 & 0 & $E(1,2) e^{j \theta_{5}}$ & $E(2,2) e^{j \theta_{5}}$ \\
\hline 0 & 0 & $C(1,1) e^{j \theta_{5}}$ & $C(2,1) e^{j \theta_{5}}$ & $E(1,1) e^{j \theta_{5}}$ & $E(2,1) e^{j \theta_{5}}$ & 0 & 0 \\
\hline 0 & 0 & $C(1,2) e^{j \theta_{5}}$ & $C(2,2) e^{j \theta_{5}}$ & $E(1,2) e^{j \theta_{5}}$ & $E(2,2) e^{j \theta_{5}}$ & 0 & 0 \\
\hline 0 & 0 & $E(1,1)$ & $E(2,1)$ & $C(1,1)$ & $C(2,1)$ & 0 & 0 \\
\hline 0 & 0 & $E(1,2)$ & $E(2,2)$ & $C(1,2)$ & $C(2,2)$ & 0 & 0 \\
\hline$E(1,1)$ & $E(2,1)$ & 0 & 0 & 0 & 0 & $C(1,1)$ & $C(2,1)$ \\
\hline$E(1,2)$ & $E(2,2)$ & 0 & 0 & 0 & 0 & $C(1,2)$ & $C(2,2)$ \\
\hline 0 & $E(1,1) e^{j \theta_{2}}$ & 0 & $E(2,1) e^{j \theta_{2}}$ & $C(1,1) e^{j \theta_{1}}$ & 0 & $C(2,1) e^{j \theta_{1}}$ & 0 \\
\hline 0 & $E(1,2) e^{j \theta_{2}}$ & 0 & $E(2,2) e^{j \theta_{2}}$ & $C(1,2) e^{j \theta_{1}}$ & 0 & $C(2,2) e^{j \theta_{1}}$ & 0 \\
\hline$E(1,1) e^{j \theta_{1}}$ & 0 & $E(2,1) e^{j \theta_{1}}$ & 0 & 0 & $C(1,1) e^{j \theta_{2}}$ & 0 & $C(2,1) e^{j \theta_{2}}$ \\
\hline$E(1,2) e^{j \theta_{1}}$ & 0 & $E(2,2) e^{j \theta_{1}}$ & 0 & 0 & $C(1,2) e^{j \theta_{2}}$ & 0 & $C(2,2) e^{j \theta_{2}}$ \\
\hline 0 & $E(1,1) e^{j \theta_{4}}$ & $E(2,1) e^{j \theta_{4}}$ & 0 & $C(1,1) e^{j \theta_{3}}$ & 0 & 0 & $C(2,1) e^{j \theta_{3}}$ \\
\hline 0 & $E(1,2) e^{j \theta_{4}}$ & $E(2,2) e^{j \theta_{4}}$ & 0 & $C(1,2) e^{j \theta_{3}}$ & 0 & 0 & $C(2,2) e^{j \theta_{3}}$ \\
\hline$E(1,1) e^{j \theta_{3}}$ & 0 & 0 & $E(2,1) e^{j \theta_{3}}$ & 0 & $C(1,1) e^{j \theta_{4}}$ & $C(2,1) e^{j \theta_{4}}$ & 0 \\
\hline$E(1,2) e^{j \theta_{3}}$ & 0 & 0 & $E(2,2) e^{j \theta_{3}}$ & 0 & $C(1,2) e^{j \theta_{4}}$ & $C(2,2) e^{j \theta_{4}}$ & 0 \\
\hline 0 & 0 & $E(1,1) e^{j \theta_{5}}$ & $\mathrm{E}(2,1) e^{j \theta_{5}}$ & $C(1,1) e^{j \theta_{5}}$ & $C(2,1) e^{j \theta_{5}}$ & 0 & 0 \\
\hline 0 & 0 & $E(1,2) e^{j \theta_{5}}$ & $E(2,2) e^{j \theta_{5}}$ & $C(1,2) e^{j \theta_{5}}$ & $C(2,2) e^{j \theta_{5}}$ & 0 & 0 \\
\hline$E(1,1) e^{j \theta_{5}}$ & $E(2,1) e^{j \theta_{5}}$ & 0 & 0 & 0 & 0 & $C(1,1) e^{j \theta_{5}}$ & $C(2,1) e^{j \theta_{5}}$ \\
\hline$E(1,2) e^{j \theta_{5}}$ & $E(2,2) e^{j \theta_{5}}$ & 0 & 0 & 0 & 0 & $C(1,2) e^{j \theta_{5}}$ & $C(2,2) e^{j \theta_{5}}$ \\
\hline
\end{tabular}

to codeword matrices $\mathbf{C}_{m}$ and $\mathbf{C}_{p}$ transmitted from two different active antenna combinations over different symbol periods through two codeword periods are given by

$$
\begin{aligned}
& \mathbf{Y}_{m}=\sqrt{\frac{E_{s}}{N_{t}}} \mathbf{A}_{l} \mathbf{C}_{m} e^{j \theta_{l}}+\mathbf{V}_{1} \\
& \mathbf{Y}_{p}=\sqrt{\frac{E_{s}}{N_{t}}} \mathbf{A}_{f} \mathbf{C}_{p} e^{j \theta_{f}}+\mathbf{V}_{2}
\end{aligned}
$$

where the active channel matrices $\mathbf{A}_{l}$ and $\mathbf{A}_{f}$ and the angles $\theta_{l}$ and $\theta_{f}$ are related to active antenna combinations $l$ and $f$ which are different in all antenna elements.
Define matrices $\mathbf{G}\left(b_{1}, b_{2}, \ldots, b_{k}, \mathbf{C}_{m}\right)$ and $\mathbf{G}\left(b_{1}, b_{2}, \ldots, b_{k}, \mathbf{C}_{p}\right)$ as

$$
\begin{aligned}
& \mathbf{G}\left(b_{1}, b_{2}, \ldots, b_{k}, \mathbf{C}_{m}\right)=\mathbf{Y}_{m}-\sqrt{\frac{E_{s}}{N_{t}}} \mathbf{A}_{l} \mathbf{C}_{m} e^{j \theta_{l}}=\mathbf{V}_{1} \\
& \mathbf{G}\left(b_{1}, b_{2}, \ldots, b_{k}, \mathbf{C}_{p}\right)=\mathbf{Y}_{p^{-}} \sqrt{\frac{E_{s}}{N_{t}}} \mathbf{A}_{f} \mathbf{C}_{p} e^{j \theta_{f}}=\mathbf{V}_{2} \\
& \mathbf{G}\left(\tilde{b}_{1}, \tilde{b}_{2}, \ldots, \tilde{b}_{k}, \mathbf{C}_{n}\right)=\mathbf{Y}_{m}-\sqrt{\frac{E_{s}}{N_{t}}} \mathbf{A}_{l} \mathbf{C}_{n} e^{j \theta_{l}}
\end{aligned}
$$




$$
\mathbf{G}\left(\tilde{b}_{1}, \tilde{b}_{2}, \ldots, \tilde{b}_{k}, \mathbf{C}_{q}\right)=\mathbf{Y}_{p^{-}} \sqrt{\frac{E_{s}}{N_{t}}} \mathbf{A}_{\tilde{f}} \mathbf{C}_{q} e^{j \theta_{\tilde{f}}}
$$

where $\mathbf{C}_{n}$ and $\mathbf{C}_{q}$ are different than $\mathbf{C}_{m}$ and $\mathbf{C}_{p}$. The pairwise error probability of $\left(b_{1}, b_{2}, \ldots, b_{k}, \mathbf{C}_{m}, \mathbf{C}_{p}\right)$ and the decoder decided $\left(\tilde{b}_{1}, \tilde{b}_{2}, \ldots, \tilde{b}_{k}, \mathbf{C}_{n}, \mathbf{C}_{q}\right)$ is given by

$$
\begin{aligned}
& P_{r}\left(b_{1}, b_{2}, \ldots, b_{k}, \mathbf{C}_{m}, \mathbf{C}_{p} \rightarrow \tilde{b}_{1}, \tilde{b}_{2}, \ldots, \tilde{b}_{k}, \mathbf{C}_{n}, \mathbf{C}_{q}\right)= \\
& \quad P_{r}\left(\left\|\mathbf{G}\left(b_{1}, b_{2}, \ldots, b_{k}, \mathbf{C}_{m}\right)\right\|_{F}^{2}>\left\|\mathbf{G}\left(\tilde{b}_{1}, \tilde{b}_{2}, \ldots, \tilde{b}_{k}, \mathbf{C}_{n}\right)\right\|_{F}^{2}\right) \\
& \quad+P_{r}\left(\left\|\mathbf{G}\left(b_{1}, b_{2}, \ldots, b_{k}, \mathbf{C}_{p}\right)\right\|_{F}^{2}>\left\|\mathbf{G}\left(\tilde{b}_{1}, \tilde{b}_{2}, \ldots, \tilde{b}_{k}, \mathbf{C}_{q}\right)\right\|_{F}^{2}\right)
\end{aligned}
$$

where the subscript $F$ denotes the Frobenius norm of the matrix.

The mean and variance matrices of $\mathbf{G}\left(b_{1}, b_{2}, \ldots, b_{k}, \mathbf{C}_{m}\right)$ are given by

$$
\begin{gathered}
\overline{\mathbf{G}}\left(b_{1}, b_{2}, \ldots, b_{k}, \mathbf{C}_{m}\right)=E\left[\mathbf{V}_{1}\right]=\mathbf{0}_{N_{r} \times N_{t}} \\
\mathbf{\sigma}_{\mathbf{G}\left(b_{1}, b_{2}, \ldots, b_{k}, \mathbf{C}_{m}\right)}^{2}=E \mid \mathbf{G}\left(b_{1}, b_{2}, \ldots, b_{k}, \mathbf{C}_{m}\right) \\
-\left.\overline{\mathbf{G}}\left(b_{1}, b_{2}, \ldots, b_{k}, \mathbf{C}_{m}\right)\right|^{2}=N_{0} I_{N_{r} \times N_{t}}
\end{gathered}
$$

where $\mathbf{0}_{N_{r} \times N_{t}}$ is $N_{r} \times N_{t}$ zero matrix and $E$ is the expectation.

In a similar manner,

$$
\begin{gathered}
\overline{\mathbf{G}}\left(b_{1}, b_{2}, \ldots, b_{k}, \mathbf{C}_{p}\right)=\mathbf{0}_{N_{r} \times N_{t}} \\
\boldsymbol{\sigma}_{\mathbf{G}\left(b_{1}, b_{2}, \ldots, b_{k}, \mathbf{C}_{p}\right)}^{2}=N_{0} I_{N_{r} \times N_{t}}
\end{gathered}
$$

The mean and variance matrices of $\mathbf{G}\left(\tilde{b}_{1}, \tilde{b}_{2}, \ldots, \tilde{b}_{k}, \mathbf{C}_{n}\right)$ are given by

$$
\begin{aligned}
& \overline{\mathbf{G}}\left(\tilde{b}_{1}, \tilde{b}_{2}, \ldots, \tilde{b}_{k}, \mathbf{C}_{n}\right)=E\left[\mathbf{Y}_{m}-\sqrt{\frac{E_{s}}{N_{t}}} \mathbf{A}_{l} \mathbf{C}_{n} e^{j \theta_{i}}\right]=\mathbf{0}_{N_{r} \times N_{t}} \\
& \boldsymbol{\sigma}_{\mathbf{G}\left(\tilde{b}_{1}, \tilde{b}_{2}, \ldots, \tilde{b}_{k}, \mathbf{C}_{n}\right)}^{2}=E\left|\mathbf{G}\left(\tilde{b}_{1}, \tilde{b}_{2}, \ldots, \tilde{b}_{k}, \mathbf{C}_{n}\right)-\overline{\mathbf{G}}\left(\tilde{b}_{1}, \tilde{b}_{2}, \ldots, \tilde{b}_{k}, \mathbf{C}_{n}\right)\right|^{2} \\
& =E\left|\mathbf{Y}_{m^{-}} \sqrt{\frac{E_{s}}{N_{t}}} \mathbf{A}_{i} \mathbf{C}_{n} e^{i \theta_{i}}\right|^{2}
\end{aligned}
$$

$\mathbf{V}_{1}$ is independent of $\mathbf{A}_{l} \mathbf{C}_{m} e^{j \theta_{l}}$ and $\mathbf{A}_{l} \mathbf{C}_{n} e^{j \theta} \tilde{l}$, then

$$
\begin{aligned}
\boldsymbol{\sigma}_{\mathbf{G}\left(\tilde{b}_{1}, \tilde{b}_{2}, \ldots, \tilde{b}_{k}, \mathbf{C}_{n}\right)}^{2} & =E\left|\mathbf{V}_{1}\right|^{2}+\frac{E_{s}}{N_{t}} E\left(\left|\mathbf{A}_{l} \mathbf{C}_{m} e^{j \theta_{l}}-\mathbf{A}_{l} \mathbf{C}_{n} e^{j \theta_{l}}\right|^{2}\right) \\
& =N_{0} I_{N_{r} \times N_{t}}+\frac{E_{s}}{N_{t}} E\left(\left|\mathbf{A}_{l} \mathbf{C}_{m} e^{j \theta_{l}}-\mathbf{A}_{l} \mathbf{C}_{n} e^{j \theta_{l}}\right|^{2}\right)
\end{aligned}
$$

In a similar manner,

$$
\begin{gathered}
\overline{\mathbf{G}}\left(\tilde{b}_{1}, \tilde{b}_{2}, \ldots, \tilde{b}_{k}, \mathbf{C}_{q}\right)=\mathbf{0}_{N_{r} \times N_{t}} \\
\boldsymbol{\sigma}_{\mathbf{G}\left(\tilde{b}_{1}, \tilde{b}_{2}, \ldots, \tilde{b}_{k}, \mathbf{C}_{q}\right)}^{2}=N_{0} I_{N_{r} \times N_{t}}+\frac{E_{s}}{N_{t}} E\left(\left|\mathbf{A}_{f} \mathbf{C}_{p} e^{j \theta_{f}}-\mathbf{A}_{\tilde{f}} \mathbf{C}_{q} e^{j \theta_{\tilde{f}}}\right|^{2}\right)
\end{gathered}
$$

Let,

$$
\begin{array}{r}
\mathbf{D}\left(b_{1}, b_{2}, \ldots, b_{k}, \mathbf{C}_{m}\right)=\mathbf{G}\left(b_{1}, b_{2}, \ldots, b_{k}, \mathbf{C}_{m}\right) \cdot /\left(\sqrt{N_{t}} \boldsymbol{\sigma}_{\mathbf{G}\left(b_{1}, b_{2}, \ldots, b_{k}, \mathbf{C}_{m}\right)}\right) \\
(30) \\
\mathbf{D}\left(b_{1}, b_{2}, \ldots, b_{k}, \mathbf{C}_{p}\right)=\mathbf{G}\left(b_{1}, b_{2}, \ldots, b_{k}, \mathbf{C}_{p}\right) \cdot /\left(\sqrt{N_{t}} \boldsymbol{\sigma}_{\mathbf{G}\left(b_{1}, b_{2}, \ldots, b_{k}, \mathbf{C}_{p}\right)}\right)
\end{array}
$$

$$
\mathbf{D}\left(\tilde{b}_{1}, \tilde{b}_{2}, \ldots, \tilde{b}_{k}, \mathbf{C}_{n}\right)=\mathbf{G}\left(\tilde{b}_{1}, \tilde{b}_{2}, \ldots, \tilde{b}_{k}, \mathbf{C}_{n}\right) \cdot /\left(\sqrt{N_{t}} \mathbf{\sigma}_{\mathbf{G}\left(\tilde{b}_{1}, \tilde{b}_{2}, \ldots, \tilde{b}_{k}, \mathbf{C}_{n}\right)}\right)
$$

$$
\mathbf{D}\left(\tilde{b}_{1}, \tilde{b}_{2}, \ldots, \tilde{b}_{k}, \mathbf{C}_{q}\right)=\mathbf{G}\left(\tilde{b}_{1}, \tilde{b}_{2}, \ldots, \tilde{b}_{k}, \mathbf{C}_{q}\right) \cdot /\left(\sqrt{N_{t}} \boldsymbol{\sigma}_{\mathbf{G}\left(\tilde{b}_{1}, \tilde{b}_{2}, \ldots, \tilde{b}_{k}, \mathbf{C}_{q}\right)}\right)
$$

where the operation ./ is Hadamard division (element by element of the numerator and denominator matrices).

$$
\left\|\mathbf{D}\left(b_{1}, b_{2}, \ldots, b_{k}, \mathbf{C}_{m}\right)\right\|_{F}^{2}, \quad\left\|\mathbf{D}\left(b_{1}, b_{2}, \ldots, b_{k}, \mathbf{C}_{p}\right)\right\|_{F}^{2},
$$

$\left\|\mathbf{D}\left(\tilde{b}_{1}, \tilde{b}_{2}, \ldots, \tilde{b}_{k}, \mathbf{C}_{n}\right)\right\|_{F}^{2}$, and $\left\|\mathbf{D}\left(\tilde{b}_{1}, \tilde{b}_{2}, \ldots, \tilde{b}_{k}, \mathbf{C}_{q}\right)\right\|_{F}^{2}$ are central chi-square random variables with $2 N_{r}$ degrees of freedom. By using the approach in [20], the pairwise error probability can be obtained as

$$
\begin{gathered}
P_{r}\left(\left(b_{1}, b_{2}, \ldots, b_{k}, \mathbf{C}_{m}, \mathbf{C}_{p}\right) \rightarrow\left(\tilde{b}_{1}, \tilde{b}_{2}, \ldots, \tilde{b}_{k}, \mathbf{C}_{n}, \mathbf{C}_{q}\right)\right) \\
=I \frac{N_{0}}{2 N_{0}+\frac{z_{1}}{N_{t}^{2}}}\left(N_{r}, N_{r}\right)+I \frac{N_{0}}{2 N_{0}+\frac{22}{N_{t}^{2}}}\left(N_{r}, N_{r}\right)
\end{gathered}
$$

In (34), $z_{1}$ and $z_{2}$ are given by

$$
\begin{aligned}
& z_{1}=\frac{E_{s}}{N_{t}} E\left\|\mathbf{A}_{l} \mathbf{C}_{m} e^{j \theta_{l}}-\mathbf{A}_{i} \mathbf{C}_{n} e^{j \theta_{l}}\right\|_{F}^{2} \\
& z_{2}=\frac{E_{s}}{N_{t}} E\left\|\mathbf{A}_{f} \mathbf{C}_{p} e^{j \theta_{f}}-\mathbf{A}_{\tilde{f}} \mathbf{C}_{q} e^{j \theta_{f}}\right\|_{F}^{2}
\end{aligned}
$$

By using the upper bound presented in [27], the average probability of bit error is given by

$$
\begin{array}{r}
P_{b} \leq \sum_{b_{1}, \ldots, b_{k}, \mathbf{C}_{m}, \mathbf{C}_{p}} \sum_{\tilde{b}_{1}, \ldots, \tilde{b}_{k}, \mathbf{C}_{n}, \mathbf{C}_{q}} \frac{N_{e}\left(b_{1}, \ldots, b_{k}, \mathbf{C}_{m}, \mathbf{C}_{p}, \tilde{b}_{1}, \ldots, \tilde{b}_{k}, \mathbf{C}_{n}, \mathbf{C}_{q}\right)}{(k+v) 2^{k+v}} \\
\times P_{r}\left(\left(b_{1}, b_{2}, \ldots, b_{k}, \mathbf{C}_{m}, \mathbf{C}_{p}\right) \rightarrow\left(\tilde{b}_{1}, \tilde{b}_{2}, \ldots, \tilde{b}_{k}, \mathbf{C}_{n}, \mathbf{C}_{q}\right)\right)
\end{array}
$$

where $v=2 N_{t}^{2} \log _{2}(M)$ and $N_{e}\left(b_{1}, \ldots, b_{k}, \mathbf{C}_{m}, \mathbf{C}_{p}, \tilde{b}_{1}, \ldots\right.$, $\left.\tilde{b}_{k}, \mathbf{C}_{n}, \mathbf{C}_{q}\right)$ is the number of bits in error between $b_{1}, \ldots, b_{k}$, $\mathbf{C}_{m}, \mathbf{C}_{p}$ and $\tilde{b}_{1}, \ldots, \tilde{b}_{k}, \mathbf{C}_{n}, \mathbf{C}_{q}$. By substituting (34) in (37), 


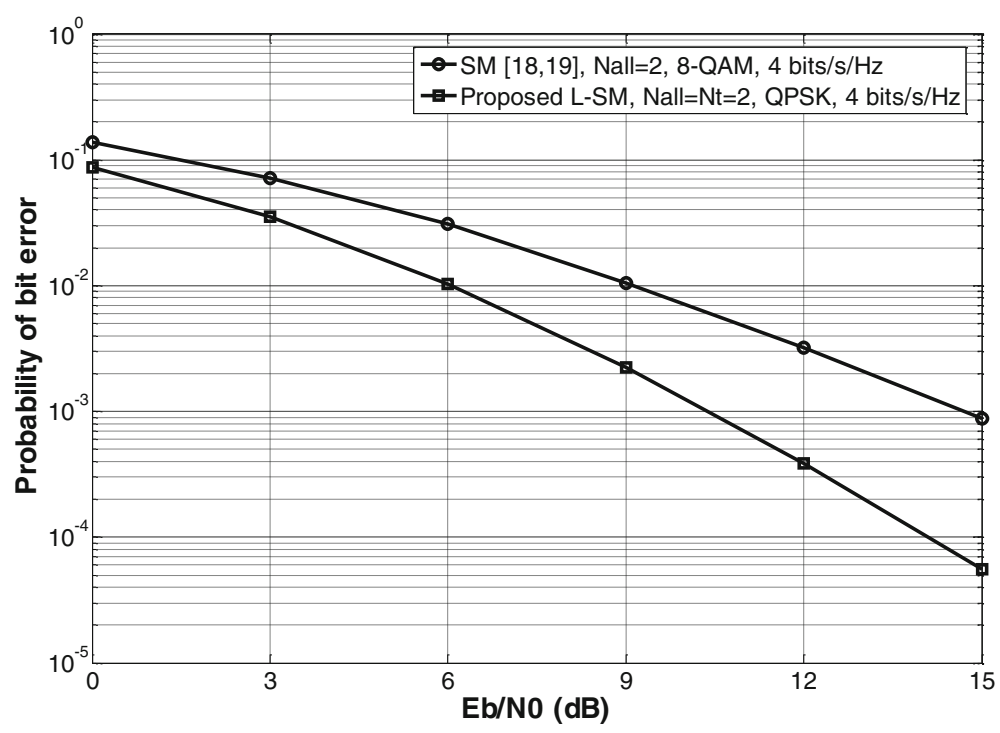

Fig. 3 Performance comparison of the SM and proposed L-SM schemes with $N_{r}=2$ for 4 bits $/ \mathrm{s} / \mathrm{Hz}$

$$
\begin{array}{r}
P_{b} \leq \sum_{b_{1}, \ldots, b_{k}, \mathbf{C}_{m}, \mathbf{C}_{p}} \sum_{\tilde{b}_{1}, \ldots, \tilde{b}_{k}, \mathbf{C}_{n}, \mathbf{C}_{q}} \frac{N_{e}\left(b_{1}, \ldots, b_{k}, \mathbf{C}_{m}, \mathbf{C}_{p}, \tilde{b}_{1}, \ldots, \tilde{b}_{k}, \mathbf{C}_{n}, \mathbf{C}_{q}\right)}{(k+v) 2^{k+v}} \\
\times\left(I_{\frac{N_{0}}{2 N_{0}+\frac{z_{1}}{N_{t}^{2}}}}\left(N_{r}, N_{r}\right)+I_{\frac{N_{0}}{2 N_{0}+\frac{22}{N_{t}^{2}}}}\left(N_{r}, N_{r}\right)\right)
\end{array}
$$

\section{Numerical results and comparisons}

In this section, we compare the performance of the proposed L-SM scheme with the spatial modulation schemes: SM [18, 19], STBC-SM [21], STBC-CSM [23], and STBC-TSM [25] with the same number of antennas and same spectral efficiency.

Figure 3 shows the bit error probability of the proposed L-SM scheme with two layers compared to SM scheme $[18,19]$ as a function of $E_{b} / N_{0}$ where $E_{b}$ is the bit energy. The proposed L-SM and SM schemes are equipped with two transmit and two receive antennas. For the proposed L-SM scheme $N_{\text {all }}=N_{t}=2$ and the codeword is transmitted over one codeword period without any antenna selection. QPSK and 8-QAM modulation are used for the proposed L-SM and SM schemes, respectively. This yields spectral efficiency of $4 \mathrm{bits} / \mathrm{s} / \mathrm{Hz}$ for both of them. Example 1 in Section 4.2 explains the

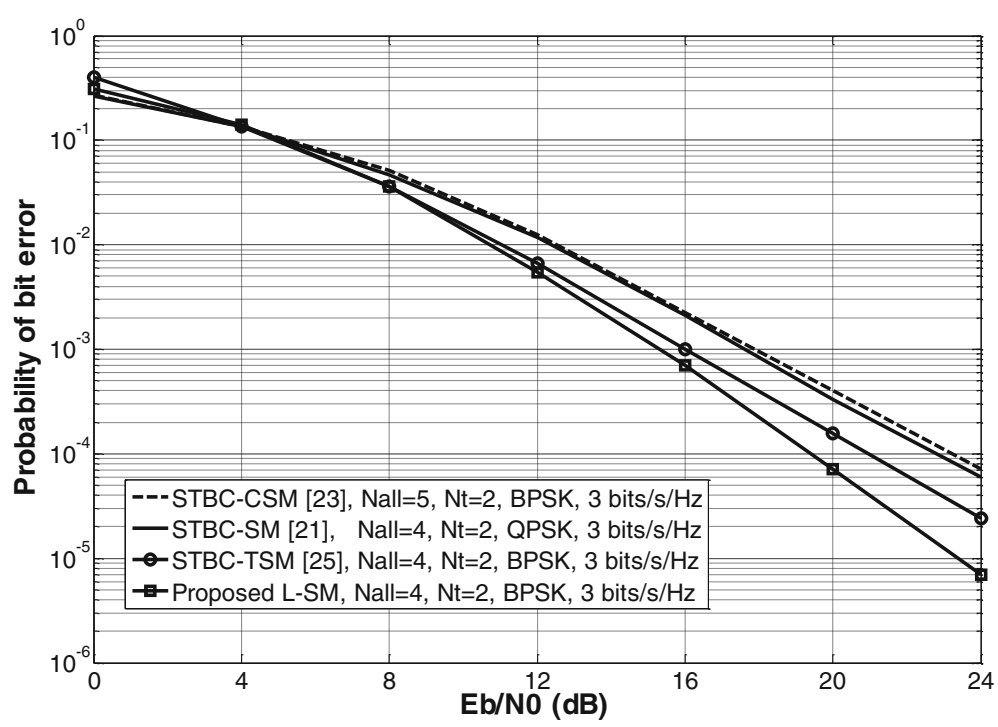

Fig. 4 Performance comparison of STBC-CSM, STBC-SM, STBC-TSM, and proposed L-SM schemes with $N_{r}=1$ for 3 bits $/ \mathrm{s} / \mathrm{Hz}$ 


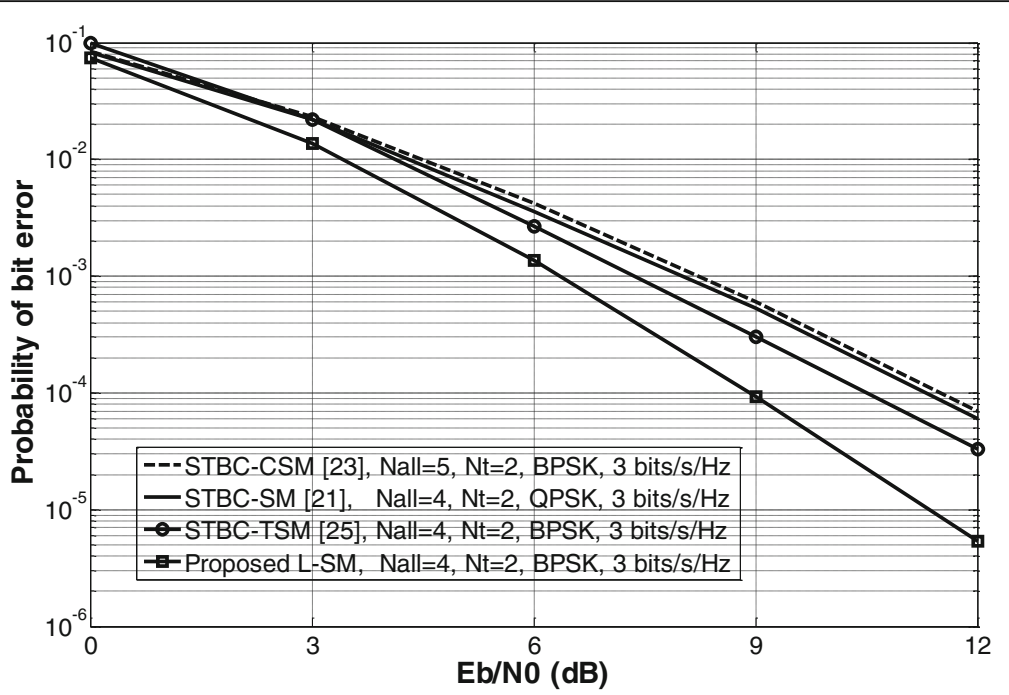

Fig. 5 Performance comparison of STBC-CSM, STBC-SM, STBC-TSM, and proposed L-SM schemes with $N_{r}=2$ for 3 bits $/ \mathrm{s} / \mathrm{Hz}$

proposed scheme for this case. As can be observed from the figure, the proposed scheme significantly outperforms the SM scheme.

In Figs. 4 and 5, the performance of the proposed L-SM, STBC-SM, STBC-CSM and STBC-TSM schemes is presented for $N_{r}=1$ and $N_{r}=2$. All these schemes except STBC-CSM are equipped with four transmit antennas, and the SM-CSM scheme is equipped with five transmit antennas. The number of active transmit antennas of all these schemes is equal to 2. To achieve spectral efficiency of $3 \mathrm{bits} / \mathrm{s} / \mathrm{Hz}$ for all schemes, BPSK modulation is used for the proposed L-SM, STBC-CSM, and STBC-TSM schemes and QPSK is used for
STBC-SM scheme. The proposed L-SM scheme in this case is explained by example 2 of Section 4.2. In both figures, significant improvement in the performance of the proposed L-SM scheme is achieved compared to that of the all other schemes.

Figure 6 further compares the performance of the proposed L-SM scheme with SM scheme. Both schemes use eight transmit and two receive antennas. The proposed L-SM scheme uses two active transmit antennas and QPSK modulation. The total number of antenna selections $N=\frac{2 \times 8 !}{(8-2) ! 2 !}+8=64$, but in this example, we use only 16 antenna selections to produce $k=4$ which yields spectral efficiency of $5 \mathrm{bits} / \mathrm{s} / \mathrm{Hz}$. The $\mathrm{SM}$ scheme uses

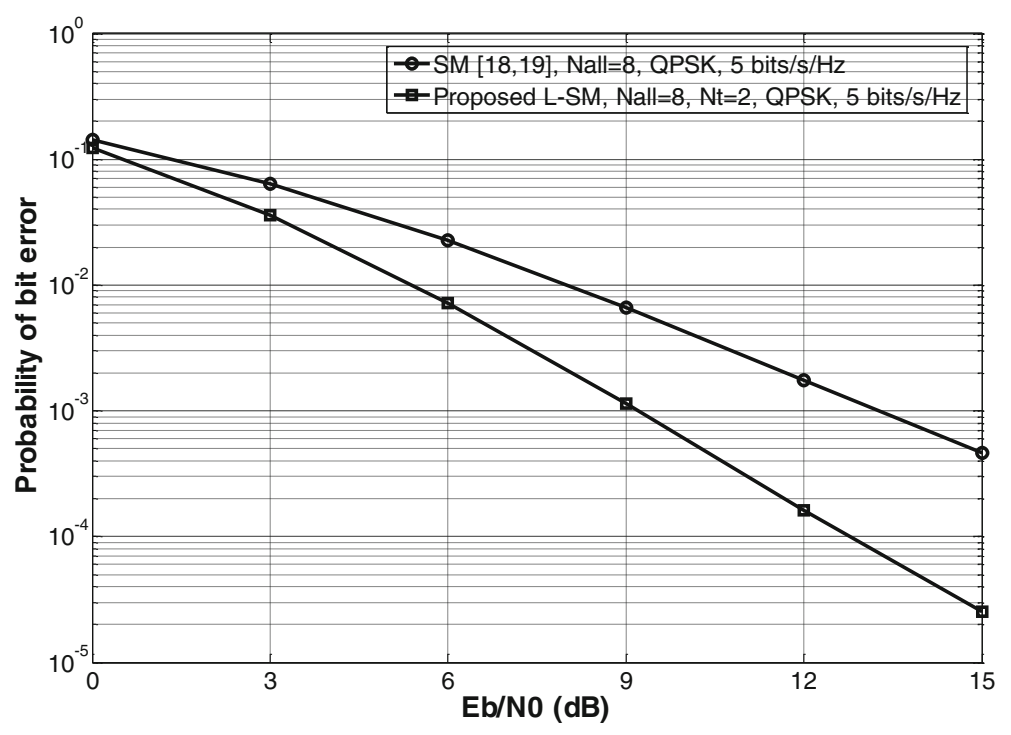

Fig. 6 Performance comparison of SM and proposed L-SM schemes with $N_{r}=2$ for 5 bits $/ \mathrm{s} / \mathrm{Hz}$ 
QPSK modulation to satisfy the same spectral efficiency of $5 \mathrm{bits} / \mathrm{s} / \mathrm{Hz}$. As expected, similar behavior as in the case of Fig. 3 is observed.

Note that the generalized spatial modulation scheme in [20] uses more than one active transmit antenna at each time period to increase the spectral efficiency. The performance of this scheme is slightly smaller than the performance of the spatial modulation scheme in [18, 19] with the same spectral efficiency as explained in [20]. Therefore, no need to compare this scheme with our scheme since our scheme significantly outperforms the spatial modulation scheme in $[18,19]$. Also, no need to compare our scheme with the H-STBC-SM [22] and CIOD-SM-H [24] schemes because their performance is less than that of the STBC-CSM [23] and STBC-TSM [25] schemes with the same transmit antennas and same spectral efficiency as can be shown in $[23,25]$.

\section{Conclusions}

We have proposed L-SM scheme with high performance compared to all existing spatial modulation schemes. The proposed L-SM scheme uses a number of layers with the same number of active transmit antennas. At each antenna selection, the proposed L-SM scheme maximizes the diversity and coding gains. The performance of the proposed L-SM scheme was analyzed. Numerical results of the proposed L-SM and different spatial modulation schemes were presented. Significant improvement in the performance of the proposed L-SM scheme is achieved compared to that of the other schemes.

\begin{abstract}
Abbreviations
BPSK: Binary phase shift keying; CIOD-SM-H: Complex interleaved orthogonal design SM with the high degree of spatial modulation; $E_{b} / N_{0}$ : Bit energy to noise ratio; H-STBC-SM: High-rate space-time block coded spatial modulation; L-SM: Layered spatial modulation; MIMO: Multiple input multiple output; M-QAM: M-ary quadrature amplitude modulation; QPSK: Quadrature phase shift keying; SM: Spatial modulation; STBC: Space-time block coding; STBC-CSM: Spatial modulation space-time block coding scheme with cyclic structure; STBC-

SM: Space-time block coded spatial modulation; STBC-TSM: Cyclic temporally and spatially modulated space-time block coding
\end{abstract}

\section{Authors' contributions}

All contributions are from the sole author SA. The author read and approved the final manuscript.

\section{Competing interests}

The author declares that he has no competing interests.

\section{Publisher's Note}

Springer Nature remains neutral with regard to jurisdictional claims in published maps and institutional affiliations.

Received: 25 April 2018 Accepted: 18 September 2018

Published online: 01 October 2018

\section{References}

1. G. Foshini, M. Gans, On the limits of wireless communications in a fading environment when using multiple antennas. Wireless Personal

Communications 6(3), 311-335 (1998)
2. E. Telatar, Capacity of multi-antenna Gaussian channels. Eur Trans Telecom 10(6), 585-595 (1999)

3. V. Tarokh, N. Seshadri, A. Calderbank, Space-time codes for high data rate wireless communication: performance criterion and code construction. IEEE Trans Inform Theory 44(2), 744-765 (1998)

4. V. Tarokh, A. Naguib, N. Seshadri, A.R. Calderbank, Space-time codes for high data rate wireless communication: performance criteria in the presence of channel estimation errors, mobility, and multiple paths. IEEE Trans. on Communications 47(2), 199-207 (1999)

5. V. Tarokh, H. Jafarkhani, A. Calderbank, Space-time block codes from orthogonal designs. IEEE Trans. Inform. Theory 45(5), 1456-1467 (1999)

6. S.M. Alamouti, A simple transmit diversity technique for wireless communications. IEEE J Select Areas Commun 16(8), 1451-1458 (1998)

7. S. Mudulodu, A. Paulraj, A transmit diversity scheme for frequency-selective fading channels (Paper presented at the 2000 IEEE Global Telecommunications Conference, San Francisco, 2000), pp. 1089-1093 2

8. A. Ahmadi, S. Talebi, M. Shahabinejad, A new approach to fast decode quasi-orthogonal space-time block codes. IEEE Transact Wireless Commun 14(1), 165-176 (2015)

9. V. Jiménez, A. Gameiro, A. Armada, Space-time code diversity by phase rotation in multi-carrier multi-user systems. EURASIP J Wireless Commun Net 2013(1), 1687-1499 (2013)

10. G. Foschini, Layered space-time architecture for wireless communication in a fading environment when using multiple antennas. Bell Labs Tech J 1(2), 41-59 (1996)

11. G. Foschini, G. Golden, R. Valenzuela, P. Wolniansky, Simplified processing for high spectral efficiency wireless communication employing multielement arrays. IEEE J. Select. Areas Commun 17(11), 1841-1852 (1999)

12. B. Hassibi, B.M. Hochwald, High-rate codes that are linear in space and time. IEEE Trans. Inform. Theory 48(7), 1804-1824 (2002)

13. R. Heath Jr., A. Paulraj, Linear dispersion codes for MIMO systems based on frame theory. IEEE Trans. Signal Processing 50(10), 2429-2441 (2002)

14. X. Wang, S. Alkhawaldeh, Y. Shayan, A new approach to diversity and multiplexing gains for wideband MIMO channels. IEEE Transaction Wireless Commun 6(1), 90-100 (2007)

15. X. Gao, Z. Wu, Precoded spatial multiplexing MIMO system with spatial component interleaver. EURASIP J Wireless Commun Netw 2016(64), 1-15 (2016)

16. J. Lee, J.Y. Lee, Y.H. Lee, Spatial multiplexing of OFDM signals with QPSK modulation over ESPAR. IEEE Transactions on Vehicular Technology 66(6), 4914-4923 (2017)

17. C. Cheng, H. Sari, S. Sezginer, Y. Su, Enhanced spatial multiplexing — a novel approach to MIMO signal design (Paper presented in the 2016 IEEE International Conference on Communications (ICC), Kuala Lumpur, 2016), pp. 22-27

18. R. Mesleh, H. Haas, S. Sinanovi'c, C. Ahn, S. Yun, Spatial modulation. IEEE Trans Veh Technol 57(4), 2228-2241 ((2008))

19. J. Jeganathan, A. Ghrayeb, L. Szczecinski, Spatial modulation: optimal detection and performance analysis. IEEE Communications Letters 12(8), 545-547 (2008)

20. A. Younis, N. Serafimovski, R. Mesleh, H. Haas, Generalized spatial modulation (Paper presented in the 2010Conference Record of the Forty Fourth Asilomar Conference on Signals, Systems and Computers, Pacific Grove, 2010), pp. 7-10

21. E. Basar, U. Aygolu, E. Panayirci, H.V. Poor, Space-time block coded spatial modulation. IEEE Trans. Commun 59(3), 823-832 (2011)

22. M.T. Le, V.D. Ngo, H.A. Mai, X.N. Tran, High-rate space-time block coded spatial modulation (Paper presented in the 2012 International Conference on Advanced Technologies for Communications (ATC 2012), Hanoi, 2012), pp. 278-282

23. X. Li, L. Wang, High rate space-time block coded spatial modulation with cyclic structure. IEEE Commun Letters 18(4), 532-535 (2014)

24. R. Rajashekar, K.V.S. Hari, Modulation diversity for spatial modulation using complex interleaved orthogonal design (Paper presented in the Proc. TENCON IEEE Region Conf, Cebu, 2012), pp. 1-6

25. A.G. Helmy, M. Di Renzo, N. Al-Dhahir, Enhanced-reliability cyclic generalized spatial-and-temporal modulation. IEEE Communications Letters 20(12), 2374-2377 (2016)

26. Y. Viniotis, Probability and random processes for electrical engineers (WCB/ McGraw-Hill, Boston, 1988)

27. J. Proakis, Digital Communications, 4th edn. (McGraw-Hill, New York, 2001) 